\title{
Activation of extracytoplasmic function sigma factors upon removal of glucolipids and reduction of phosphatidylglycerol content in Bacillus subtilis cells lacking lipoteichoic acid
}

\author{
Takahiro Seki ${ }^{\dagger}$, Takuya Furumi, Michihiro Hashimoto, \\ Hiroshi Hara and Satoshi Matsuoka* \\ Department of Biochemistry and Molecular Biology, Graduate School of Science and Engineering, \\ Saitama University, 255 Shimo-Okubo, Sakura-ku, Saitama, Saitama 338-8570, Japan
}

(Received 30 August 2018, accepted 2 November 2018; J-STAGE Advance published date: 9 April 2019)

In Bacillus subtilis, extracytoplasmic function (ECF) sigma factors are activated by reduction of phosphatidylglycerol (PG) content, absence of glucolipids, or absence of lipoteichoic acid (LTA). LTA is synthesized by polymerization of the glycerophosphate moiety of PG onto diglucosyldiacylglycerol (DGlcDG), a major glucolipid in B. subtilis, in the plasma membrane. Thus, reduction of PG content or absence of glucolipids might cause some changes in LTA, and hence we investigated whether reduction of PG content or absence of glucolipids induces the activation of ECF sigma factors independently from an ensuing change in LTA. Disruption of ugtP, responsible for glucolipid synthesis, in cells lacking LTA caused an additive increase of activation levels of $\sigma^{\mathrm{M}}, \sigma^{\mathrm{X}}, \sigma^{\mathrm{V}}$ and $\sigma^{\mathrm{Y}}$ (3.1-, 2.2-, 2.1- and 1.4-fold, respectively), relative to their activation levels in cells lacking LTA alone. Reduction of PG content (by repressing $\mathrm{P}_{\text {spac }}-p g s A$ ) in the cells lacking LTA caused an additive increase of activation levels of $\sigma^{\mathrm{M}}, \sigma^{\mathrm{W}}$ and $\sigma^{\mathrm{V}}(2.3-, 1.9$ - and 2.2-fold, respectively). These results suggested that absence of glucolipids or reduction of PG alone, not the possible secondary alteration in LTA, leads to changes that affect the regulation systems of some ECF sigma factors in the plasma membrane.

Key words: ECF sigma factor, lipoteichoic acid, glucolipids, phosphatidylglycerol, lipid composition

\section{INTRODUCTION}

Bacillus subtilis has seven extracytoplasmic function (ECF) sigma factors, $\sigma^{\mathrm{M}}, \sigma^{\mathrm{V}}, \sigma^{\mathrm{W}}, \sigma^{\mathrm{X}}, \sigma^{\mathrm{Y}}, \sigma^{\mathrm{Ylac}}$ and $\sigma^{\mathrm{Z}}$, which are a group of bacterial sigma factors that direct transcription of genes involved in tasks such as maintenance of cells upon external stresses. Except $\sigma^{Z}$, these are regulated directly by their respective cognate transmembrane anti- $\sigma$ factors, which sequester the $\sigma$ factors and keep them inactive. Under stress conditions the $\sigma$ factors are released from the anti- $\sigma$ factors, form RNA polymerase holoenzymes and transcribe the respective regulon genes. The genes encoding $\sigma$ and anti- $\sigma$ factors form operons whose transcription is autoregulated. Acti-

Edited by Kei Asai

* Corresponding author. E-mail: matsuoka@mail.saitama-u.ac.jp

$\dagger$ Present address: Department of Applied Chemistry and Biotechnology, Faculty of Engineering, Chiba University, 1-33

Yayoi-cho, Inage-ku, Chiba, Chiba 263-8522, Japan

DOI: http://doi.org/10.1266/ggs.18-00046 vation of ECF sigma factors can result from a number of stresses on the cell envelope. For example, $\sigma^{\mathrm{M}}$ is activated in response to high salt, cell wall antibiotics, ethanol, heat, acid and superoxide stresses (Thackray and Moir, 2003; Asai et al., 2005; Luo and Helmann, 2012); $\sigma^{\mathrm{V}}$ is strongly and specifically activated in response to lysozyme (Guariglia-Oropeza and Helmann, 2011; Ho et al., 2011); $\sigma^{\mathrm{W}}$ is activated in response to salt, alkali, antibiotics inhibiting cell wall biosynthesis and oxidative stresses (Petersohn et al., 2001; Helmann, 2002; Cao et al., 2003; Asai et al., 2005; Butcher and Helmann, 2006); and $\sigma^{\mathrm{x}}$ is activated by inhibitors of peptidoglycan and wall teichoic acid (WTA) biosynthesis, and serves to alter cell envelope properties to protect against cationic antimicrobial peptides (Helmann, 2002; Cao and Helmann, 2004; Murray and Stanley-Wall, 2010). Among these ECF sigma factors, it is clear that $\sigma^{\mathrm{V}}$ and $\sigma^{\mathrm{W}}$ are activated by proteolysis of anti-sigma factors via regulated intramembrane proteolysis (Schobel et al., 2004; Zellmeier et al., 2006; Heinrich et al., 2009; Hastie et al., 2013, 2014, 2016). 
As shown in Fig. 1, B. subtilis membranes contain various lipids, including glucosylated diacylglycerol, monoglucosyldiacylglycerol (MGlcDG), diglucosyldiacylglycerol (DGlcDG) and triglucosyldiacylglycerol (TDGlcDG), and a complex glycerophospholipid, lysylphosphatidylglycerol (LysylPG), in addition to the glycerophospholipids found in Escherichia coli membranes, phosphatidylethanolamine (PE), phosphatidylglycerol (PG) and cardiolipin (CL) (Matsumoto et al., 2015). All genes responsible for synthesis of final lipid products are dispensable, except for pgsA, which encodes phosphatidylglycerophosphate synthase and is responsible for the committed step in PG synthesis. Lipoteichoic acid (LTA), a polymer of glycerol1-phosphate derived from PG attached to DGlcDG, by which it is anchored on the membrane, is present on the outer leaflet of the membrane (Neuhaus and Baddiley, 2003; Reichmann and Grundling, 2011).

ugtP encodes a glucosyltransferase that processively transfers glucose from UDP-glucose to diacylglycerol (Jorasch et al., 1998). Cells with a deletion of ugtP that are completely lacking glucolipids show abnormal cell morphology and activation of three ECF sigma factors, $\sigma^{\mathrm{M}}, \sigma^{\mathrm{V}}$ and $\sigma^{\mathrm{X}}$ (Salzberg and Helmann, 2008; Matsuoka et al., 2011; Hashimoto et al., 2013). The phenotype is complemented by expression of heterologous glucolipid synthase from Acholeplasma laidlawii (Matsuoka et al., 2016). Moreover, we reported that $\sigma^{\mathrm{V}}$ is activated without proteolysis of RsiV in the $\Delta u g t P$ cells (Seki et al., 2017). Cells harboring $\mathrm{P}_{\text {spac }}-p g s A$ in place of the wildtype $p g s A$ allele have a PG content reduced by half (from
$28.1 \%$ to $14.1 \%$ ), arrested cell growth, and induction of $\sigma^{\mathrm{M}}$ and $\sigma^{\mathrm{V}}$ at $60 \mathrm{~min}$ after removal of inducer (Hashimoto et al., 2009).

Bacillus subtilis has four LTA synthase genes homologous to LtaS in Staphylococcus aureus: ltaS (formerly $y f l E$ ), $y f n I, y q g S$ and $y v g J$ (Gründling and Schneewind, 2007a; Schirner et al., 2009). All LTA synthesis genes can be simultaneously deleted and therefore LTA is dispensable for B. subtilis growth (Schirner et al., 2009; Wörmann et al., 2011). Finally, six ECF sigma factors, $\sigma^{\mathrm{M}}, \sigma^{\mathrm{X}}, \sigma^{\mathrm{W}}, \sigma^{\mathrm{Ylac}}, \sigma^{\mathrm{V}}$ and $\sigma^{\mathrm{Y}}$, are induced in cells lacking LTA because of disruption of all four LTA synthase genes (Hashimoto et al., 2013). However, the exact contributions of these membrane components to the activation of ECF sigma factors in B. subtilis cells are still unclear.

In the case of $S$. aureus, deletion of $y p f P$, which is a homolog of $B$. subtilis ugtP, induces an alteration in the anchor of LTA from DGlcDG to DG (Kiriukhin et al., 2001; Gründling and Schneewind, 2007b). Therefore, it is believed that reduction of PG content and absence of glucolipids in B. subtilis cause abnormalities in LTA, as a result of which some ECF sigma factors are induced in cells lacking glucolipids or having reduced PG content. However, the pattern of activation of ECF sigma factors differs between $B$. subtilis cells with a deletion of some LTA synthase genes and those without glucolipids or with reduced PG content (Hashimoto et al., 2009, 2013). To address this difference, we investigated the effect of PG reduction and absence of glucolipids on LTA profile and activity of ECF sigma factors in this study.

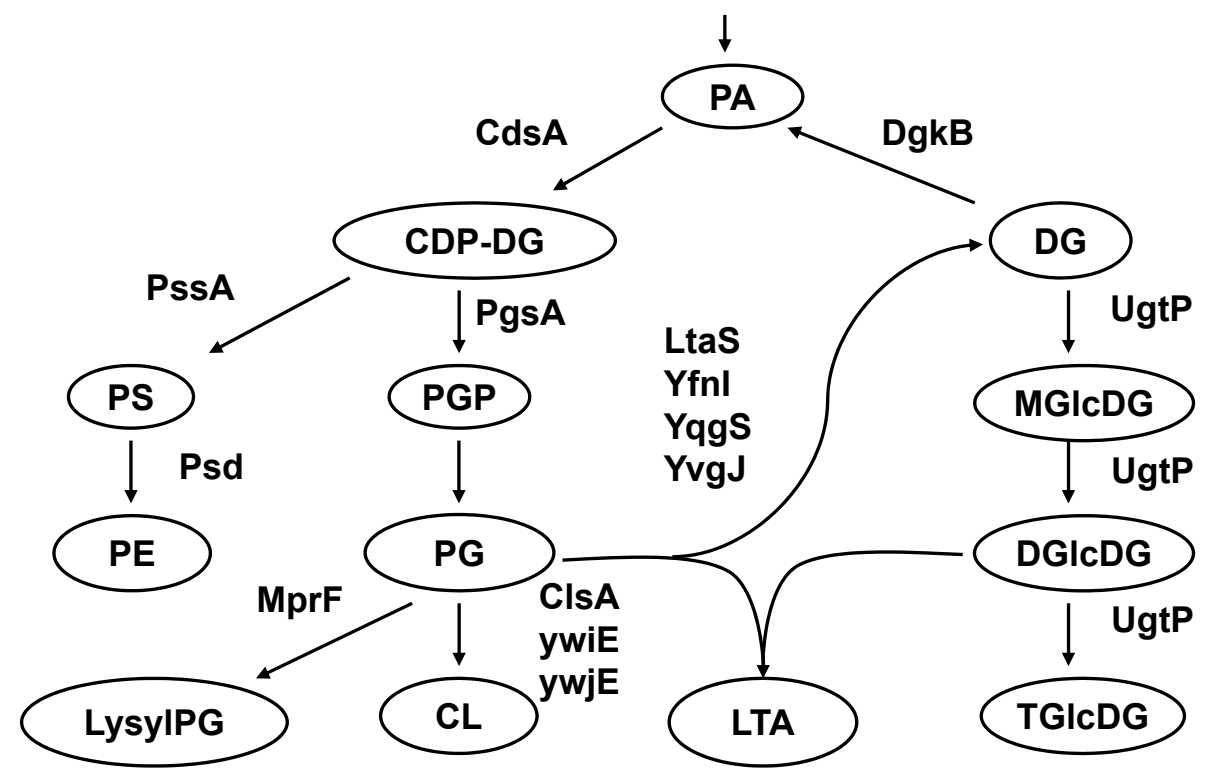

Fig. 1. Biosynthetic pathways for phospholipids in B. subtilis. The compiled pathways are from Matsumoto et al. (2015). The gene product catalyzing each step is indicated. PG, Phosphatidylglycerol; PE, phosphatidylethanolamine; CL, cardiolipin; lysylPG, lysylphosphatidylglycerol; PA, phosphatidic acid; CDP-DG, CDP-diacylglycerol; MGlcDG, monoglucosyldiacylglycerol; DGlcDG, diglucosyldiacylglycerol; TGlcDG, triglucosyldiacylglycerol; DG, diacylglycerol; LTA, lipoteichoic acid. 


\section{MATERIALS AND METHODS}

Bacterial strains, media and bacterial growth The B. subtilis and E. coli strains and plasmids used in this study are listed in Table 1. Luria-Bertani (LB) medium contained $1 \%$ tryptone (Difco), $0.5 \%$ yeast extract (Difco) and $1 \% \mathrm{NaCl}$. Synthetic media CI and CII were used for competence development for transformation of B. subtilis cells (Anagnostopoulos and Crawford, 1961). When required, the following supplements were added to the media $\left(\mathrm{ml}^{-1}\right)$ : 50 or $100 \mu \mathrm{g}$ spectinomycin (Sigma), $0.5 \mu \mathrm{g}$ erythromycin (Sigma), $10 \mu \mathrm{g}$ neomycin (Wako), $5 \mu \mathrm{g}$ chloramphenicol (Sigma), $5 \mu \mathrm{g}$ tetracycline (Sigma) or $100 \mu \mathrm{g}$ ampicillin (Wako). Isopropyl- $\beta-D-1-$ thiogalactopyranoside (IPTG) (Sigma) was added at a final concentration of $1 \mathrm{mM}$ when required. Cells were grown at $37{ }^{\circ} \mathrm{C}$, and growth was monitored by measuring turbidity with a TAITEC photometer, miniphoto518R (530 nm filter). For solid media, 1.5\% agar (Difco) was included.
Genetic and recombinant DNA procedures These were based on standard methods (Miller, 1992; Sambrook and Russell, 2001).

SDS-PAGE and immunoblot analysis of LTA Cells of 168 (wild-type), YTB019 ( $\Delta u g t P)$ and SLD08 ( $\Delta l t a S$ $\Delta y f n I \Delta y q g S \Delta y v g J)$ were grown to $\mathrm{OD}_{530}=0.5$ at $37{ }^{\circ} \mathrm{C}$ in $5 \mathrm{ml} \mathrm{LB}$ medium and then diluted $1 / 10$ into $5 \mathrm{ml}$ of fresh $\mathrm{LB}$ medium and grown to $\mathrm{OD}_{530}=0.5$. $\mathrm{MHB} 001$ (pgsA:: $\mathrm{P}_{\text {spac }}-$ pgsA) cells were cultivated overnight at $37^{\circ} \mathrm{C}$ in $5 \mathrm{ml} \mathrm{LB}$ medium containing $1 \mathrm{mM}$ IPTG and then washed three times using fresh LB medium without IPTG and diluted to $\mathrm{OD}_{530}=0.05$ into $5 \mathrm{ml}$ fresh $\mathrm{LB}$ medium without IPTG. Cells were grown to the point at which turbidity stopped increasing $\left(\mathrm{OD}_{530}=0.5\right)$ and $5 \mathrm{ml}$ of culture was collected. Bacterial pellets were suspended in $2 \times$ protein sample buffer normalized for $\mathrm{OD}_{530}$ readings; that is, $25 \mu \mathrm{l}$ of $2 \times$ sample buffer was used per ml culture of $\mathrm{OD}_{530}=0.5$. Samples were boiled for $45 \mathrm{~min}$, centrifuged at $19,000 \mathrm{~g}$ for $5 \mathrm{~min}$ at $4{ }^{\circ} \mathrm{C}$, and $10 \mu \mathrm{l}$ of the supernatant was loaded on a $15 \%$ Extra PAGE One Precast Gel

Table 1. Bacterial strains and plasmids used in this study

\begin{tabular}{|c|c|c|}
\hline Strain & Genotype & Source \\
\hline \multicolumn{3}{|l|}{ B. subtilis } \\
\hline 168 & $\operatorname{trpC2}$ & Laboratory stock \\
\hline YTB019 & $168 \Delta u g t P:: k a n$ & Matsuoka et al., 2011 \\
\hline MHB001 & 168 pgsA:: $\mathrm{P}_{\text {spac }}-p g s A$ erm & Hashimoto et al., 2009 \\
\hline MBS12 & $168 \Delta l t a S:: s p c \Delta y f n I:: t e t$ & Hashimoto et al., 2013 \\
\hline SLD06 & $168 \Delta l t a S:: s p c \quad \Delta y f n I:: t e t \quad \Delta y q g S$ & Hashimoto et al., 2013 \\
\hline SLD07 & $168 \Delta y f n I::$ tet $\Delta y q g S$ syvgJ & Hashimoto et al., 2013 \\
\hline SLD08 & $168 \Delta l t a S:: s p c \Delta y f n I:: t e t \Delta y q g S \Delta y v g J$ & Hashimoto et al., 2013 \\
\hline SLD09 & SLD07 $\Delta u g t P:: k a n$ & This study \\
\hline SLD10 & SLD08 $\Delta u g t P:: k a n$ & This study \\
\hline SLD11 & $\mathrm{SLD} 07$ pgsA:: $\mathrm{P}_{s p a c}-p g s A$ erm & This study \\
\hline SLD12 & $\mathrm{SLD08} \mathrm{pgsA:: \textrm {P } _ { \text { spac } } - p g s A \text { erm }}$ & This study \\
\hline \multicolumn{3}{|l|}{ E. coli } \\
\hline UE54 & $\begin{array}{l}\text { MG1655 lpp-2 } \Delta a r a 714 \text { rcsF::mini-Tn10 cam } \\
\text { pgsA::FRT-kan-FRT }\end{array}$ & Shiba et al., 2004 \\
\hline UE83 & MC4100 lpp-2 $\Delta a r a 714$ & Nagahama et al., 2006 \\
\hline FT01 & UE83 pgsA::FRT-kan-FRT & This study \\
\hline \multicolumn{3}{|l|}{ Plasmid } \\
\hline pFZY1 & F'lac replicon, lacZY bla & Koop et al., 1987 \\
\hline pFZY1- $P_{\text {sigM }}$ & $\mathrm{pFZY1} \mathrm{P}_{\text {sigm }}{ }^{\prime}-l a c Z Y$ & Seki et al., 2015 \\
\hline $\mathrm{pFZY1-P_{ \textrm {sigV } }}$ & $\mathrm{pFZY1} \mathrm{P}_{\text {sigv-lacZY }}$ & Seki et al., 2015 \\
\hline pFZY1-P ${ }_{\text {sigx }}{ }^{\prime}$ & $\mathrm{pFZY} 1 \mathrm{P}_{s i g X^{\prime}}-l a c Z Y$ & Seki et al., 2015 \\
\hline pBR322 & bla tet & Balbás et al., 1986 \\
\hline pBR-sigM-anti-sigM & pBR322 $\mathrm{P}_{\text {sigM }}{ }^{\prime}-s i g M-y h d L-y h d K$ tet & Seki et al., 2015 \\
\hline pBR-sigV-anti-sigV & pBR322 $\mathrm{P}_{\text {sigV-sigV-rsiV tet }}$ & Seki et al., 2015 \\
\hline pBR-sigX-anti-sigX & pBR322 $\mathrm{P}_{\text {sigX }}{ }^{\prime}-s i g X-r s i X$ tet & Seki et al., 2015 \\
\hline
\end{tabular}


(Nacalai Tesque). LTA-specific primary antibody (clone 55; HyCult Biotechnology) and peroxidase-conjugated AffiniPure goat anti-mouse IgG secondary antibody (Jackson ImmunoResearch Laboratories) were used at a dilution of 1:1000 for LTA detection. Incubation with antibody was performed in TBS-T buffer containing $2 \%$ BSA (Sigma). Luminata Forte Western HRP substrate (Millipore) was used for immunodetection in Western blotting experiments according to the manufacturer's instructions. Western analysis bands were detected by a C-DiGit scanner (LI-COR) and signal intensities were analyzed by ImageStudio (LI-COR).

Real-time RT-PCR Cells of 168, YTB019, SLD08, SLD10 ( $\Delta l t a S \Delta y f n I \Delta y q g S \Delta y v g J \Delta u g t P)$, MHB001 and SLD12 ( $\operatorname{ltaS} \Delta y f n I$ syqgS $\Delta y v g J$ pgsA::Pspac-pgsA) were grown under the same conditions described above for the SDS-PAGE experiments. A 500- $\mu$ l aliquot of each sample was collected, and RNA was then extracted using a Total RNA Purification Kit (Jena Bioscience) and an RNasefree DNase set (Roche Applied Science) according to the manufacturer's recommendations. The DNase-treated RNA was reverse-transcribed using ReverTra Ace qPCR RT Master Mix with gDNA Remover (TOYOBO) according to the manufacturer's recommendations. Real-time RT-PCR with SYBR Green I was performed using Thunderbird SYBR qPCR Mix (TOYOBO). Real-time RTPCR was carried out as described previously, using the pairs of primers for $\operatorname{sig} A, \operatorname{sig} M, \operatorname{sig} V, \operatorname{sig} W, \operatorname{sig} X, \operatorname{sig} Y$ and $y l a C$ listed in Supplementary Table S1 (Hashimoto et al., 2013). Each real-time RT-PCR was performed in at least quintuplicate with $\operatorname{sig} A$ as the internal standard.

Lipid analysis $\left[1-{ }^{14} \mathrm{C}\right]$-labeled membrane lipids of 168, YTB019, SLD08, SLD10, MHB001 and SLD12 were obtained as previously described (Hashimoto et al., 2009, 2013). Lipid fractions were subjected to $2 \mathrm{D}$ thin layer chromatography on silica gel (no. 60, Merck); the first dimension was with chloroform/methanol/water (65:25:4, by volume) and the second was with chloroform/methanol/ acetic acid (65:25:10, by volume) as described previously (Kawai et al., 2004). Spots for $\left[{ }^{14} \mathrm{C}\right]$-labeled lipids were visualized and quantified with an FLA-7000 bioimaging analyzer (Fujifilm). The molar percentage of each component (evaluated from the relative number of carbon atoms) was calculated.

$\boldsymbol{\beta}$-galactosidase assay in $\boldsymbol{E}$. coli The $E$. coli cells with plasmids were grown to mid-log phase and 1-ml aliquots of the culture were withdrawn, and $\beta$-galactosidase activity in the cells was assayed as described previously (Wang and Doi, 1984).

\section{RESULTS AND DISCUSSION}

Lack of glucolipids affects the structure of LTA To reveal the effect of an absence of glucolipids and reduction of PG content on the LTA profile in B. subtilis, cell extracts from a mid-log phase culture $\left(\mathrm{OD}_{530}=0.5\right)$ of wild-type cells (168 strain, hereafter called WT cells), cells lacking glucolipids (YTB019 strain, hereafter called $\Delta$ GLs cells), cells with a reduced PG content (MHB001 strain, hereafter called $\mathrm{PG}^{\text {Red. }}$ cells) and cells lacking LTA (SLD08 strain, hereafter called $\Delta$ LTA cells) were prepared and LTA content analyzed by Western blot. A signal in the $15-\mathrm{kDa}$ area was detected in WT cells but not in $\triangle$ LTA cells (Fig. 2), indicating that it is an LTAspecific signal. In $\Delta$ GLs cells the signal was shifted to the 20-kDa area (Fig. 2). This reduced LTA migration on SDS-PAGE observed in $B$. subtilis cells with a deletion of ugtP suggests that ugtP deletion induces the alteration of the LTA anchor from DGlcDG to DG, as seen in the case of the $S$. aureus ypfP deletion (Gründling and Schneewind, 2006). However, no change of signal pattern was observed in $\mathrm{PG}^{\text {Red. }}$ cells. These results suggest that not the reduction of PG, but the absence of DGlcDG affected the LTA profile. In addition, a broad signal

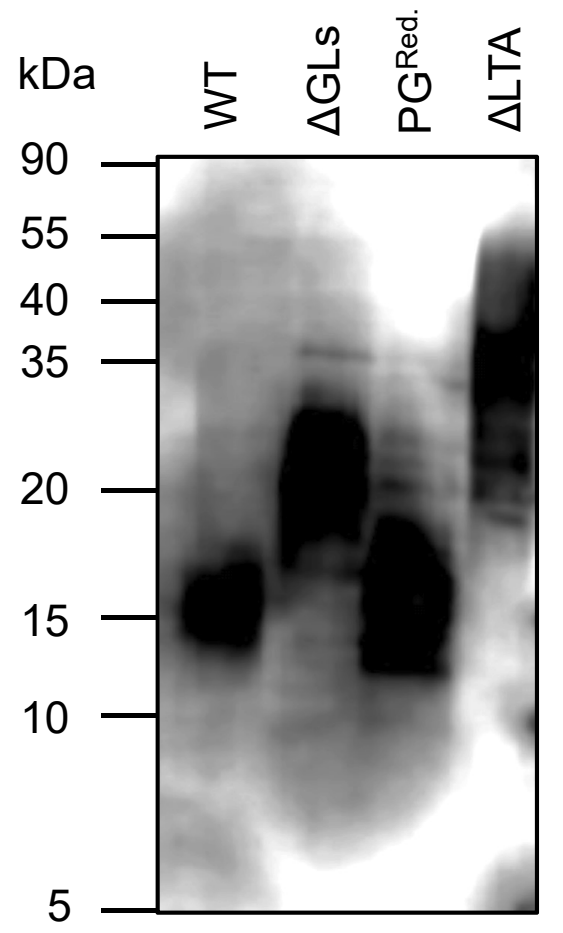

Fig. 2. LTA detection by Western blotting. LTA was extracted from B. subtilis strains 168 (wild-type; WT), YTB019 ( $\Delta u g t P$; $\Delta \mathrm{GLs}$ ), MHB001 (pgsA:: $\mathrm{P}_{\text {spac }}-$ pgsA; $\mathrm{PG}^{\text {Red. }}$ ) and SLD08 ( $\Delta l t a S$ $\Delta y f n I \quad \Delta y q g S \quad \Delta y v g J ; \Delta \mathrm{LTA})$. The samples were normalized based on culture $\mathrm{OD}_{530}$ values, separated by $15 \%$ SDS-PAGE, and detected with an LTA-specific antibody by C-DiGit scanner. The positions of protein molecular mass markers (in $\mathrm{kDa}$ ) are indicated on the left. 
around the $35-\mathrm{kDa}$ area, which is a WTA signal caused by LTA antibody cross-reacting with WTA (Wörmann et al., 2011), was observed in $\Delta$ LTA cells (Fig. 2). Although the signal around $35 \mathrm{kDa}$ was not observed in WT cells or $\mathrm{PG}^{\text {Red. }}$ cells, a weak signal was detected in $\Delta \mathrm{GLs}$ cells. The change of LTA profile as well as the lack of LTA may reflect an increase of the WTA content to compensate for the LTA abnormality.

Lack of glucolipids activates ECF sigma factors independent of abnormalities in LTA To determine whether or not the lack of glucolipids activates ECF sigma factors independent of the change in LTA profile observed in $\Delta$ GLs cells (Fig. 2), we deleted $u g t P$ from $\Delta$ LTA cells and analyzed the activation levels of ECF sigma factors. The mRNA level of ECF sigma factors was regarded as the activation level, because the transcription of each ECF sigma factor is autoregulated. The mRNA levels of the genes for six ECF sigma factors in $\Delta$ GLs cells, $\triangle$ LTA cells and cells lacking both LTA and glucolipids (SLD10 strain, hereafter called $\Delta$ LTA $\Delta$ GLs cells) were determined by real-time RT-PCR to estimate the activation levels of sigma factors. In $\Delta \mathrm{GLs}$ cells the mRNA levels of the four ECF sigma factor genes $\operatorname{sig} M, \operatorname{sig} X, \operatorname{sig} V$ and $\operatorname{sig} Y$ were increased 2.3-, 1.5-, 2.7- and 1.5-fold, respectively, compared with those of WT cells (Fig. 3). In this work a novel activation of $\sigma^{\mathrm{Y}}$ was observed. In $\Delta$ LTA cells the mRNA levels of sig $M, \operatorname{sig} X, \operatorname{sig} W, y l a C, \operatorname{sig} V$ and $\operatorname{sig} Y$ were increased (Fig. 3 ). In $\Delta$ LTA $\Delta$ GLs cells the mRNA levels of $\operatorname{sig} M, \operatorname{sig} X$, $\operatorname{sig} V$ and $\operatorname{sig} Y$ were increased 4.5-, 1.6-, 4.1- and 3.7-fold, respectively, compared with those of WT cells, but the levels of sigW and $y l a C$ were not changed (Fig. 3). This result shows that the effects of a lack of both glucolipids and LTA on the activation of three sigma factors ( $\operatorname{sig} M, \operatorname{sig} V$ and $\operatorname{sig} Y$ ) were additive in $\Delta$ GLs $\triangle$ LTA cells, suggesting that the removal of glucolipids activates the three ECF sigma factors $\sigma^{\mathrm{M}}, \sigma^{\mathrm{V}}$ and $\sigma^{\mathrm{Y}}$ independent of the absence of LTA.

To investigate whether the additive increase of activation levels of $\sigma^{\mathrm{M}}, \sigma^{\mathrm{V}}$ and $\sigma^{\mathrm{Y}}$ observed in $\Delta \mathrm{LTA} \Delta \mathrm{GLs}$ cells was caused by a secondary effect on the lipid composition induced by simultaneous deletion of the ugtP and LTA synthase genes, the lipid composition of cells lacking glucolipids and/or LTA was examined (Table 2). In $\triangle$ LTA cells, CL, CDP-DG and DG were accumulated (2.3-, 6.9and 2.8-fold, respectively), and DGlcDG and LysylPG were reduced (4.0- and 1.5-fold) compared with their content in WT cells. In $\Delta \mathrm{GLs}$ cells, the CL content of $\Delta \mathrm{GLs}$ cells was increased (2.7-fold) over WT cells and CDP-DG content was decreased $(8.0$-fold). In $\Delta \mathrm{LTA} \Delta \mathrm{GLs}$ cells, the CDP-

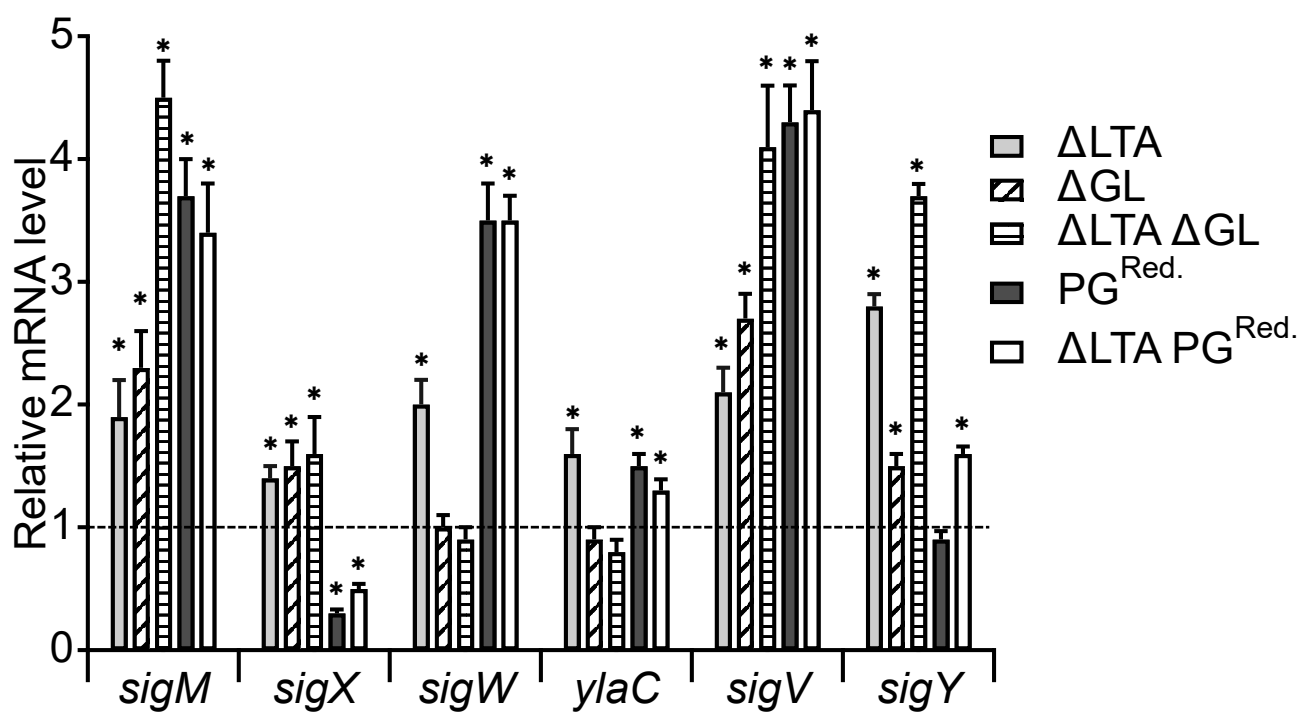

Fig. 3. Activation levels of ECF sigma factors in B. subtilis. Strains used were B. subtilis 168 (wild-type; WT) and its derivatives, SLD08 ( $\Delta l t a S \Delta y f n I \Delta y q g S \Delta y v g J ; \Delta \mathrm{LTA})$, YTB019 ( $\Delta u g t P ; \Delta \mathrm{GLs),} \mathrm{SLD10} \mathrm{(} \Delta l t a S \Delta y f n I \Delta y q g S \Delta y v g J$

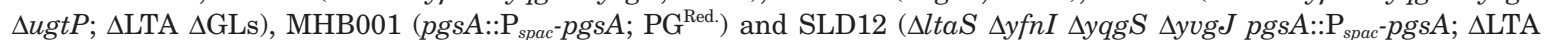
$\mathrm{PG}^{\text {Red.). }}$ WT cells, $\Delta \mathrm{LTA}$ cells, $\Delta \mathrm{GL}$ cells and $\Delta \mathrm{LTA} \Delta \mathrm{GLs}$ cells were cultivated in LB medium to exponential growth phase $\left(\mathrm{OD}_{530}=0.5\right)$ and total RNA was extracted and used for preparation of cDNA samples. Cultures of $\mathrm{PG}^{\mathrm{Red}}$. cells and $\triangle \mathrm{LTA} \mathrm{PG}^{\text {Red. }}$ cells were grown in LB medium supplemented with $1 \mathrm{mM}$ IPTG to exponential growth phase, transferred into fresh medium and cultivated without IPTG to the point at which turbidity reached an $\mathrm{OD}_{530}$ of 0.5 , and then total RNA was extracted and used for preparation of cDNA samples. The cDNA samples were then subjected to real-time RT-PCR analysis with the appropriate primer pairs for $\operatorname{sig} M, \operatorname{sig} V, \operatorname{sig} W, \operatorname{sig} X, \operatorname{sig} Y$ and $y \operatorname{laC}$, with the primer pair for sigA as the internal control. Mean values $\pm \mathrm{SD}$, calculated from six measurements excluding the highest and lowest values obtained from eight PCR assays, of the level of each sigma factor mRNA in the mutant cells, relative to those of WT cells (dotted line), are presented. Asterisks indicate significant differences as determined by Student's $t$-test: $* P<0.01$. 
Table 2. Lipid compositions of mutants

\begin{tabular}{|c|c|c|c|c|c|c|c|c|c|c|c|}
\hline \multicolumn{12}{|c|}{$\%$ of total lipid } \\
\hline Strain & $\mathrm{PG}$ & $\mathrm{PE}$ & CL & LysylPG & $\mathrm{PA}$ & CDP-DG & MGlcDG & DGlcDG & TGlcDG & DG & Others \\
\hline 168 & $2 \pm 7.7$ & $.8 \pm 2.7$ & $3 \pm 3.9$ & $11.6 \pm 0.5$ & $9 \pm 0.2$ & $1.6 \pm 0.4$ & $1.2 \pm 0.7$ & $8.0 \pm 0.7$ & 0.4 & $4.9 \pm 0.8$ & $2.1 \pm 0.7$ \\
\hline$\Delta \mathrm{LTA}$ & $20.9 \pm 2.3$ & $16.2 \pm 1.2$ & $19.2 \pm 7.5$ & $7.6 \pm 0.5$ & $2.7 \pm 2.5$ & $11.0 \pm 3.2$ & $0.6 \pm 0.2$ & $2.0 \pm 0.5$ & $0.6 \pm 0.3$ & $13.8 \pm 3.2$ & $5.4 \pm 2.8$ \\
\hline$\Delta \mathrm{GLs}$ & $26.7 \pm 4.2$ & $21.1 \pm 1.7$ & $22.4 \pm 8.4$ & $12.8 \pm 0.7$ & $1.9 \pm 0.6$ & $0.2 \pm 0.04$ & N.D. & N.D. & N.D. & $7.6 \pm 1.5$ & $7.4 \pm$ \\
\hline$\Delta \mathrm{LTA} \Delta \mathrm{GLs}$ & $35.4 \pm 6.5$ & $18.0 \pm 1.4$ & $9.5 \pm 3.2$ & $13.5 \pm 1.4$ & $4.6 \pm 2.1$ & $1.6 \pm 0.4$ & N.D. & N.D. & N.D. & $14.8 \pm 1.7$ & $2.7 \pm 1.7$ \\
\hline $\mathrm{PG}^{\text {Red. }}$ & $3.4 \pm 1.0$ & $47.6 \pm 1.8$ & $3.7 \pm 0.4$ & $6.8 \pm 0.9$ & $3.5 \pm 0.7$ & $2.1 \pm 0.3$ & $0.6 \pm 0.4$ & $6.8 \pm 0.8$ & $0.1 \pm 0.05$ & $19.2 \pm 0.6$ & $6.2 \pm 0.4$ \\
\hline$\Delta \mathrm{LTA} \mathrm{PG}^{\mathrm{Red}}$ & $5.7 \pm 0.7$ & $38.9 \pm 2.2$ & $6.6 \pm 3.1$ & $4.4 \pm 0.9$ & $2.5 \pm 0.2$ & $7.1 \pm 1.7$ & $0.6 \pm 0.1$ & $2.5 \pm 1.1$ & $0.2 \pm 0.05$ & $24.7 \pm 1.6$ & $6.8 \pm 2.6$ \\
\hline
\end{tabular}

Data shown are mean \pm SD values from three different cultures. N.D.: not detected.

Table 3. Relationship between activation of ECF sigma factors and lipid composition

\begin{tabular}{|c|c|c|}
\hline Cells & ECF sigma factor & Lipid composition \\
\hline$\Delta \mathrm{LTA}$ & $\sigma^{\mathrm{M}}, \sigma^{\mathrm{X}}, \sigma^{\mathrm{W}}, \sigma^{\mathrm{YlaC}}, \sigma^{\mathrm{V}}, \sigma^{\mathrm{Y}}$ & GL (-), CL (+), CDP-DG (+), LysylPG (-), DG (+) \\
\hline$\Delta \mathrm{GL}$ & $\sigma^{\mathrm{M}}, \sigma^{\mathrm{X}}, \sigma^{\mathrm{V}}, \sigma^{\mathrm{Y}}$ & GL (N.D.), CL (+), CDP-DG (-) \\
\hline$\Delta \mathrm{LTA} \Delta \mathrm{GL}$ & $\sigma^{\mathrm{M} *}, \sigma^{\mathrm{X}}, \sigma^{\mathrm{W}}, \sigma^{\mathrm{Ylac}}, \sigma^{\mathrm{V} *}, \sigma^{\mathrm{Y} *}$ & GL (N.D.) \\
\hline $\mathrm{PG}^{\text {Red. }}$ & $\sigma^{\mathrm{M}}, \sigma^{\mathrm{W}}, \sigma^{\mathrm{YlaC}}, \sigma^{\mathrm{V}}$ & $\mathrm{PG}(-), \mathrm{PE}(+), \mathrm{CL}(-), \operatorname{LysylPG}(-), \mathrm{DG}(+)$ \\
\hline$\Delta$ LTA PG Red. & $\sigma^{\mathrm{M} * *}, \sigma^{\mathrm{W} * *}, \sigma^{\mathrm{YlaC} * *}, \sigma^{\mathrm{V} * *}, \sigma^{\mathrm{Y}}$ & PG (-), PE (+), CL (-), GL (-), CDP-DG (+), LysylPG (-), DG (+) \\
\hline
\end{tabular}

* Asterisks indicate that the ECF sigma factor was activated additively in the $\Delta$ LTA $\Delta$ GL cells compared with activation in $\triangle \mathrm{LTA}$ cells and $\Delta \mathrm{GL}$ cells.

** Double asterisks indicate that the ECF sigma factor was activated additively in the $\Delta \mathrm{LTA} \mathrm{PG}^{\text {Red. }}$ cells compared with activation in $\triangle$ LTA cells.

$(+)$ : increase; (-): decrease; N.D.: not detected.

DG content was also decreased (6.9-fold) compared with that of $\Delta$ LTA cells, indicating that $u g t P$ deletion induces the reduction of CDP-DG content. Furthermore, the CL content was decreased (2.0-fold) in $\Delta$ LTA $\Delta$ GLs cells compared with that in $\Delta$ LTA cells. Since CL was accumulated in $\Delta \mathrm{GLs}$ cells, the decrease of CL content observed in the $\Delta$ LTA $\Delta$ GLs cells was not caused by ugtP deletion. Thus, the change of CL content in the $\Delta \mathrm{LTA} \Delta \mathrm{GLS}$ cells may be caused by a secondary effect of simultaneous deletion of the ugtP and LTA synthase genes. Table 3 shows the relationship between the activated ECF sigma factors and the lipid content, which were changed in each mutant cell. In $\Delta$ LTA $\Delta$ GLs cells, only glucolipid content was changed. Nevertheless, $\sigma^{\mathrm{M}}, \sigma^{\mathrm{V}}$ and $\sigma^{\mathrm{Y}}$ were additively activated. If the change of CL and CDP-DG content activates $\sigma^{\mathrm{M}}, \sigma^{\mathrm{V}}$ and $\sigma^{\mathrm{Y}}$, the activation levels of these ECF sigma factors should be decreased in the $\triangle$ LTA $\Delta$ GLs cells, because these lipids were back to wild-type levels. However, the activation levels of $\sigma^{\mathrm{M}}, \sigma^{\mathrm{V}}$ and $\sigma^{\mathrm{Y}}$ were additively increased in the $\Delta$ LTA $\Delta$ GLs cells (Fig. 3). Therefore, the change of CL and CDP-DG content is not the cause of the activation of $\sigma^{\mathrm{M}}, \sigma^{\mathrm{V}}$ and $\sigma^{\mathrm{Y}}$.

Taken together, these results show that a lack of glucolipids activates $\sigma^{\mathrm{M}}, \sigma^{\mathrm{V}}$ and $\sigma^{\mathrm{Y}}$ independent of the changes in LTA structure and other lipid composition caused by deletion of ugtP. The results also suggest that $\sigma^{\mathrm{M}}, \sigma^{\mathrm{V}}$ and $\sigma^{\mathrm{Y}}$ sensed the lack of glucolipids and of LTA as distinct stresses, but that $\sigma^{\mathrm{X}}$ sensed them as a single stress. Although there are no reports that a lack of glucolipids is induced by environmental stresses in B. subtilis, we found that glucolipid content decreased when $B$. subtilis cells were grown in high salt condition (S. Matsuoka, unpublished results). Therefore, the membrane of cells without glucolipids may be mimicking that of cells exposed to high salt stress.

\section{Reduction of phosphatidylglycerol content induces ECF sigma factors independent of a possible change} in LTA The reduction of $\mathrm{PG}$ content by restriction of pgsA expression did not result in a change of LTA profile (Fig. 2). This suggests the possibility that reduction of PG content directly induces ECF sigma factors irrespective of LTA. To determine whether the reduction of PG content can induce the ECF sigma factors in the absence of LTA, pgsA expression in $\Delta$ LTA cells was restricted and we analyzed the activation levels of ECF sigma factors in the $\triangle$ LTA cells with a reduced PG content (SLD12 strain, hereafter called $\triangle \mathrm{LTA} \mathrm{PG}^{\text {Red. }}$ cells). The mRNA levels of the genes for six ECF sigma factors in $\mathrm{PG}^{\mathrm{Red}}$ and $\triangle \mathrm{LTA}$ $\mathrm{PG}^{\text {Red. }}$ cells were determined by real-time RT-PCR to estimate the activation levels of the six sigma factors. WT cells were used as a control for comparison of mRNA levels. In $\mathrm{PG}^{\text {Red. }}$ cells the levels of mRNA from the four ECF sigma factor genes $\operatorname{sig} M$, sig $W, y l a C$ and $\operatorname{sig} V$ were increased 3.7-, 3.5-, 1.5- and 4.5-fold, respectively, compared with those of WT cells (Fig. 3). In this work the 
activation of $\sigma^{\mathrm{W}}$ and $\sigma^{\mathrm{YlaC}}$ was observed in addition to $\sigma^{\mathrm{M}}$ and $\sigma^{\mathrm{V}}$. Moreover, the mRNA level of $\operatorname{sig} X$ was decreased 3.3-fold in $\mathrm{PG}^{\text {Red. }}$ cells. In $\triangle \mathrm{LTA} \mathrm{PG}^{\text {Red. }}$ cells the mRNA levels of $\operatorname{sig} M$, $\operatorname{sig} W, y l a C$, $\operatorname{sig} V$ and $\operatorname{sig} Y$ were increased 3.4-, 3.5-, 1.3-, 4.4- and 1.6-fold, respectively, compared with those of WT cells, but the level of $\operatorname{sig} X$ was decreased 2.0-fold (Fig. 3). The activation levels of $\sigma^{\mathrm{M}}, \sigma^{\mathrm{W}}$ and $\sigma^{\mathrm{V}}$ in $\triangle$ LTA PG ${ }^{\text {Red. }}$ cells were higher than those in $\triangle$ LTA cells, indicating that the reduction of $\mathrm{PG}$ content activated these ECF sigma factors even without LTA. Therefore, these results suggest that the reduction of $\mathrm{PG}$ content induces $\sigma^{\mathrm{M}}, \sigma^{\mathrm{W}}$ and $\sigma^{\mathrm{V}}$ directly.

The activation levels of four ECF sigma factors, except $\sigma^{\mathrm{X}}$ and $\sigma^{\mathrm{Y}}$, in $\triangle \mathrm{LTA} \mathrm{PG}^{\text {Red. }}$ cells corresponded with those in $\mathrm{PG}^{\text {Red. }}$ cells (Fig. 3 ). To test whether the correspondence of the activation levels of the four ECF sigma factors $\left(\sigma^{\mathrm{M}}\right.$, $\sigma^{\mathrm{W}}, \sigma^{\mathrm{YlaC}}$ and $\sigma^{\mathrm{V}}$ ) between $\Delta \mathrm{LTA} \mathrm{PG}^{\text {Red. }}$ cells and $\mathrm{PG}^{\text {Red. }}$ cells was caused by insufficient reduction of $\mathrm{PG}$ content in $\triangle$ LTA PG $^{\text {Red. }}$ cells, the lipid composition of $\mathrm{PG}^{\text {Red. }}$ cells and $\triangle \mathrm{LTA} \mathrm{PG}^{\text {Red. }}$ cells was examined (Table 2 ). In $\mathrm{PG}^{\text {Red. }}$ cells CL and lysylPG, which are positioned downstream of PG in the lipid biosynthesis pathway (Fig. 1), were also reduced (2.2- and 1.7-fold, respectively) in addition to $\mathrm{PG}$ (10.6-fold) compared with their levels in WT cells. Moreover, the $\mathrm{PE}$ and $\mathrm{DG}$ content of $\mathrm{PG}^{\text {Red. }}$ cells was increased (2.0- and 3.9-fold, respectively). In $\triangle \mathrm{LTA} \mathrm{PG}^{\text {Red. }}$ cells $\mathrm{PG}$, CL and lysylPG levels were decreased (3.7-, 2.9- and 1.7fold, respectively) and PE and DG levels were increased (2.4- and 1.8-fold, respectively) compared with those in $\Delta$ LTA cells. The reduction level of PG compared to that in WT cells (6.4-fold) in $\Delta \mathrm{LTA} \mathrm{PG}^{\text {Red. }}$ cells was comparable with that seen in $\mathrm{PG}^{\text {Red. }}$ cells. This indicates that the correspondence of the activation levels of the four ECF sigma factors between $\triangle \mathrm{LTA} \mathrm{PG}^{\text {Red. }}$ cells and $\mathrm{PG}^{\text {Red. }}$ cells was not caused by insufficient reduction of the PG content of $\triangle \mathrm{LTA} \mathrm{PG}^{\text {Red. }}$ cells. Table 3 shows that in $\triangle \mathrm{LTA}$ $\mathrm{PG}^{\text {Red. }}$ cells, the effects of deletion of LTA synthase genes and restriction of $p g s A$ expression on lipid composition were additive. Nevertheless, the effects of deleting LTA synthase genes on activation levels of $\sigma^{\mathrm{M}}, \sigma^{\mathrm{W}}, \sigma^{\mathrm{YlaC}}$ and $\sigma^{\mathrm{V}}$ were masked. Therefore, the correspondence suggests that the activation mechanisms of $\sigma^{\mathrm{M}}, \sigma^{\mathrm{W}}, \sigma^{\mathrm{YlaC}}$ and $\sigma^{\mathrm{V}}$ sensed the reduction of PG content and lack of LTA as the same stress. As in the case of $E$. coli LacY lactose permease, proper distribution of negative charge on the cell membrane may be required for folding and function of anti-sigma factors (Dowhan and Bogdanov, 2009; Bogdanov et al., 2010). The effect of a lack of LTA on membrane negative charge density may be weak enough to be masked by the reduction of PG content, because LTA, which is localized to the outer leaflet of the cell membrane through its linkage to DGlcDG, is farther from the membrane than $\mathrm{PG}$, which is a component of the membrane. Hence, the effect of the absence of LTA on activities of ECF sigma factors in $\triangle \mathrm{LTA} \mathrm{PG}^{\text {Red. }}$ cells may be masked owing to the reduction in PG levels. Since PG is an essential lipid molecule for B. subtilis growth, significant reduction of $\mathrm{PG}$ content is not induced by environmental stress. On the other hand, the PG content of a daptomycin-resistant strain is reportedly low, similar to that of $\mathrm{PG}^{\text {Red. }}$ cells (Hachmann et al., 2011). $\sigma^{\mathrm{M}}$ and $\sigma^{\mathrm{W}}$ are also activated in the daptomycin-resistant strain, suggesting that these ECF sigma factors are activated by a reduction of $\mathrm{PG}$ content.
A

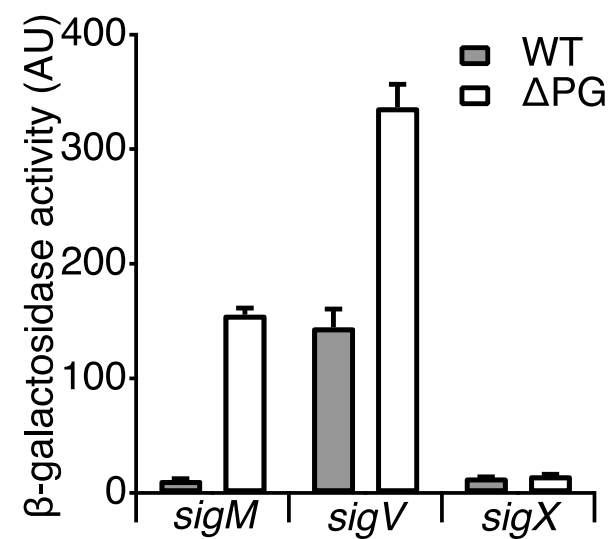

B

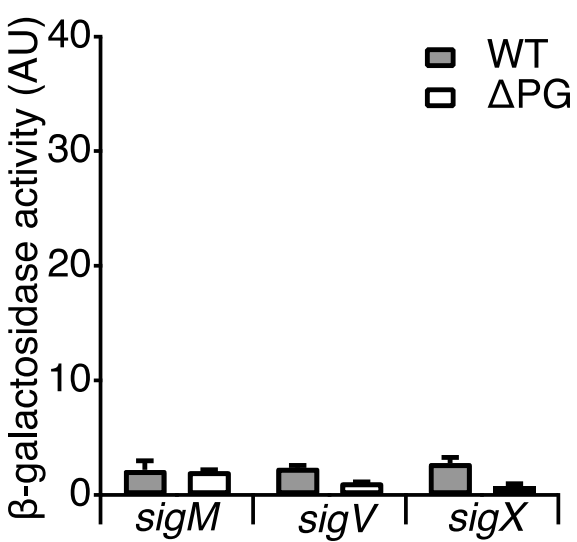

Fig. 4. Effect of activities of $\sigma^{\mathrm{M}}, \sigma^{\mathrm{V}}$ and $\sigma^{\mathrm{X}}$ on PG using an $E$. coli heterologous expression system. (A) $E$. coli UE83 (WT) and its derivative strain FT01 ( $p g s A ; \Delta \mathrm{PG})$, harboring pFZY1-P sigM $_{\text {- }}$ and pBR-sigM-antisigM (sigM operon), pFZY1- $\mathrm{P}_{\text {sigV }}$ and pBR-sigV-rsiV (sigV operon), pFZY1- $\mathrm{P}_{\text {sigX }}$ and pBR-sigX-rsiX (sigX operon), were used. (B) UE83 (WT) and FT01 $(\Delta \mathrm{PG})$, harboring pFZY1- $\mathrm{P}_{\text {sigm }}(\operatorname{sig} M)$, pFZY1- $\mathrm{P}_{\text {sigv }}(\operatorname{sigV})$ or pFZY1-P $\mathrm{P}_{\text {sigX }}(\operatorname{sigX}$ ), and pBR322 (void) were used as control assays. Overnight cultures were diluted 100-fold in $\mathrm{LB}$ medium and cultivated with shaking at $30{ }^{\circ} \mathrm{C}$. At $\mathrm{OD}_{530}=0.3$ on the miniphoto518R photometer, $\beta$-galactosidase activity was measured. The means and standard errors of three measurements are shown. 

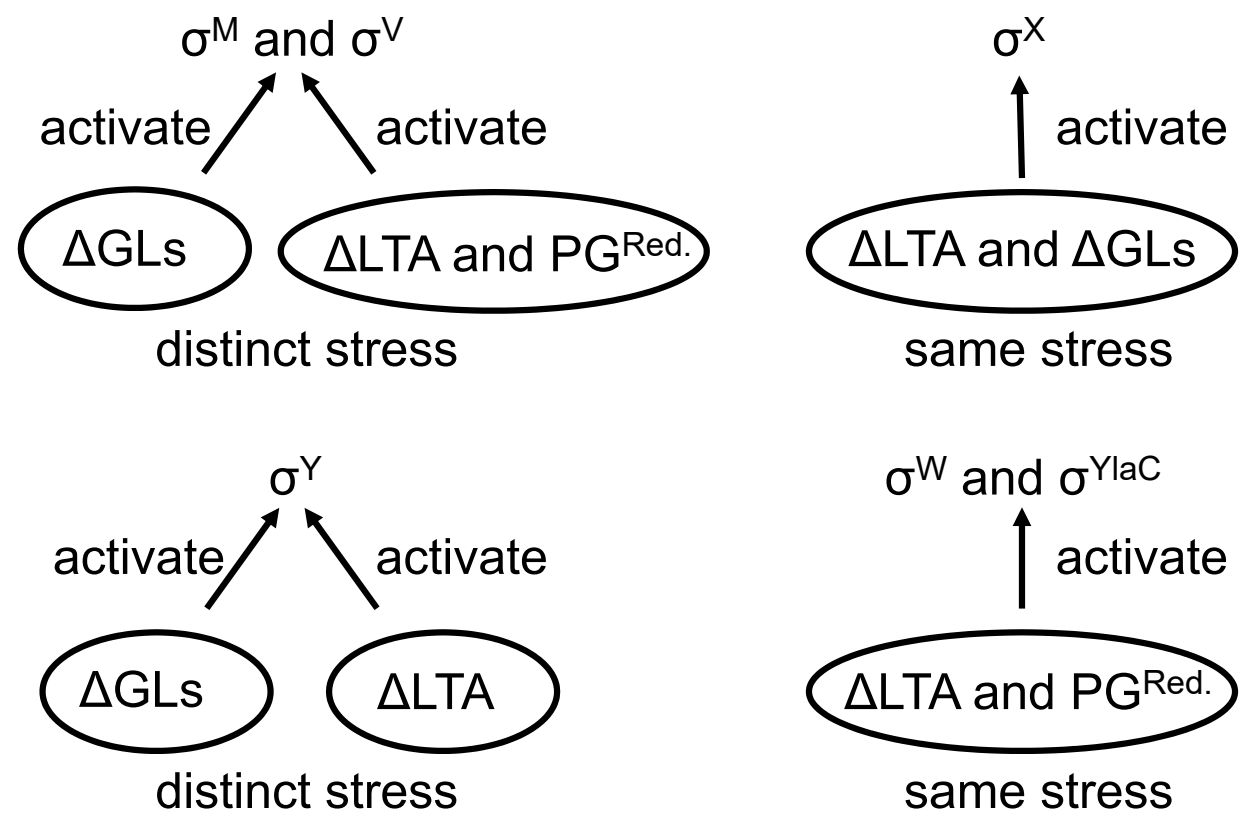

Fig. 5. Summary of relationship between activation of ECF sigma factors and changes of lipid composition. Lack of GLs $(\triangle \mathrm{GLs})$ and lack of lipoteichoic acid $(\triangle \mathrm{LTA})$ activated $\sigma^{\mathrm{M}}$ and $\sigma^{\mathrm{V}}$ independently, whereas $\triangle \mathrm{LTA}$ and reduction of $\mathrm{PG}$ content $\left(\mathrm{PG}^{\text {Red. }}\right.$ ) were not distinguishable in their activation of $\sigma^{\mathrm{M}}$ and $\sigma^{\mathrm{V}}$. Therefore, $\Delta \mathrm{GLs}$ and $\mathrm{PG} \mathrm{Ged}^{\text {Red }} \Delta \mathrm{LTA}$ activate $\sigma^{\mathrm{M}}$ and $\sigma^{\mathrm{V}}$ as distinct stresses. $\sigma^{\mathrm{Y}}$ was activated by $\Delta \mathrm{GLs}$ and $\Delta \mathrm{LTA}$ independently but not activated by $\mathrm{PG}^{\text {Red. }}$. $\quad \sigma^{\mathrm{X}}$ was not additively activated by $\Delta \mathrm{GLs}$ and $\Delta$ LTA. Hence, these activate $\sigma^{\mathrm{X}}$ as the same stress. $\Delta$ LTA and $\mathrm{PG}^{\text {Red. }}$ were not distinguishable in their activation of $\sigma^{\mathrm{W}}$ and $\sigma^{\mathrm{YlaC}}$. Hence, these activate $\sigma^{\mathrm{W}}$ and $\sigma^{\mathrm{YlaC}}$ as the same stress.

Induction of $\sigma^{\mathrm{M}}$ and $\sigma^{\mathrm{V}}$ in $E$. coli cells lacking PG Escherichia coli can lack PG due to deletion of pgsA (Kikuchi et al., 2000; Suzuki et al., 2002). To confirm that the activities of $\sigma^{\mathrm{M}}, \sigma^{\mathrm{V}}$ and $\sigma^{\mathrm{X}}$ were altered by the absence of PG, the activation levels of $\sigma^{\mathrm{M}}, \sigma^{\mathrm{V}}$ and $\sigma^{\mathrm{x}}$ were examined using an $E$. coli heterologous expression system. We deleted the $p g s A$ gene in a strain with a defect in lpp and introduced two plasmids: one was a pBR322 derivative having an operon of an ECF sigma factor and anti-sigma factor (either sigM-yhdLK, sigV-rsiV or sigX-rsiX) and the other an $\mathrm{F}^{\prime}$ plasmid having a transcriptional fusion of a promoter recognized by an ECF sigma factor and lac $Z$ $\left(\mathrm{P}_{\text {sig }} M^{\prime}-l a c Z, \mathrm{P}_{\text {sigv }}\right.$-lac $Z$ or $\left.\mathrm{P}_{\text {sig } X^{\prime}-l a c Z}\right)$ as a reporter, allowing us to monitor $\beta$-galactosidase activity as a proxy for the induction levels of $\sigma^{\mathrm{M}}, \sigma^{\mathrm{V}}$ and $\sigma^{\mathrm{x}}$. In the $\Delta p g s A$ cells the activities of $\sigma^{\mathrm{M}}$ and $\sigma^{\mathrm{V}}$ were significantly increased, by 13.7- and 2.3-fold, respectively, compared with those in WT cells (Fig. 4A), but were not increased in $\Delta p g s A$ cells lacking the sigM-yhdLK or sigV-rsiV operons (Fig. 4B), indicating that $\sigma^{\mathrm{M}}$ and $\sigma^{\mathrm{V}}$ are induced by the absence of PG in E. coli cells. These results support our hypothesis that the reduction of $\mathrm{PG}$ content activates $\sigma^{\mathrm{M}}$ and $\sigma^{\mathrm{V}}$ independent of the absence of LTA in $\triangle \mathrm{LTA} \mathrm{PG}^{\text {Red. }}$ cells of $B$. subtilis (Fig. 3). Unexpectedly, the $\operatorname{sig} X-r s i X$ operon did not work properly in E. coli $\triangle p g s A$ cells (Fig. 4A).

\section{CONCLUSION}

In this study, we found that the deletion of ugtP additively activated $\sigma^{\mathrm{M}}, \sigma^{\mathrm{V}}$ and $\sigma^{\mathrm{Y}}$ in cells lacking LTA, and that the restriction of $p g s A$ expression also additively activated $\sigma^{\mathrm{M}}, \sigma^{\mathrm{V}}$ and, $\sigma^{\mathrm{W}}$ (Fig. 3). Moreover, $\sigma^{\mathrm{M}}$ and $\sigma^{\mathrm{V}}$ responded directly to a lack of PG in E. coli as well as a lack of glucolipids (Fig. 4A) (see also Seki et al., 2015). Therefore, we concluded that a lack of glucolipids and a reduction of PG content directly activate some ECF sigma factors independently of LTA disorder in $B$. subtilis. Our data also suggested that $\sigma^{\mathrm{M}}$ and $\sigma^{\mathrm{V}}$ can sense $\Delta$ GLs and PG ${ }^{\text {red. }}-\Delta$ LTA as distinct stresses; $\sigma^{\mathrm{X}}$ can sense $\Delta \mathrm{GLs}-\Delta \mathrm{LTA}$ and $\mathrm{PG}^{\text {Red. }}-\Delta \mathrm{LTA}$ as distinct stresses; $\sigma^{\mathrm{Y}}$ can sense $\Delta \mathrm{GLs}$ and $\Delta \mathrm{LTA}$ as distinct stresses; $\sigma^{\mathrm{W}}$ and $\sigma^{\mathrm{YlaC}}$ can sense $\mathrm{PG}^{\text {Red. }}-\Delta \mathrm{LTA}$; and $\sigma^{\mathrm{X}}$ can sense $\Delta \mathrm{GLs}-\Delta \mathrm{LTA}$ (Fig. 5). This implies that these molecules have specific or overlapping functions on the membrane or cell surface. To determine the exact function of each lipid and LTA in the cells, further analysis of the activation mechanisms of each of the ECF sigma factors will be necessary.

We are grateful to K. C. Burtis for carefully reading the manuscript. T. S. is a research fellow of the Japan Society for the Promotion of Science (JSPS). This work was supported in part by JSPS KAKENHI (Grant-in-Aid for JSPS Research Fellows) Grant Numbers JP16J10629 to T. S. and 15K18664 to S. M. 


\section{CONFLICTS OF INTEREST}

None declared.

\section{REFERENCES}

Anagnostopoulos, C., and Crawford, I. P. (1961) Transformation studies on the linkage of markers in the tryptophan pathway in Bacillus subtilis. Proc. Natl. Acad. Sci. USA 47, 378-390.

Asai, K., Matsumoto, T., and Sadaie, Y. (2005) ECF (extracytoplasmic function) Sigma factors of Bacillus subtilis. In: Survival and Death in Bacteria. (ed.: Yamada, M), pp. 143-153. Research Signpost, Kerala, India.

Bogdanov, M., Heacock, P., Guan, Z., and Dowhan, W. (2010) Plasticity of lipid-protein interactions in the function and topogenesis of the membrane protein lactose permease from Escherichia coli. Proc. Natl. Acad. Sci. USA 107, 1505715062.

Butcher, B. G., and Helmann, J. D. (2006) Identification of Bacillus subtilis $\sigma^{\mathrm{W}}$-dependent genes that provide intrinsic resistance to antimicrobial compounds produced by Bacilli. Mol. Microbiol. 60, 765-782.

Cao, M., and Helmann, J. D. (2004) The Bacillus subtilis extracytoplasmic-function $\sigma^{\mathrm{X}}$ factor regulates modification of the cell envelope and resistance to cationic antimicrobial peptides. J. Bacteriol. 186, 1136-1146.

Cao, M., Salzberg, L., Tsai, C. S., Mascher, T., Bonilla, C., Wang, T., Ye, R. W., Márquez-Magaña, L., and Helmann, J. D. (2003) Regulation of the Bacillus subtilis extracytoplasmic function protein $\sigma^{\mathrm{Y}}$ and its target promoters. J. Bacteriol. 185, 4883-4890.

Dowhan, W., and Bogdanov, M. (2009) Lipid-dependent membrane protein topogenesis. Annu. Rev. Biochem. 78, 515540 .

Gründling, A., and Schneewind, O. (2006) Cross-linked peptidoglycan mediates lysostaphin binding to the cell wall envelope of Staphylococcus aureus. J. Bacteriol. 188, 24632472.

Gründling, A., and Schneewind, O. (2007a) Genes required for glycolipid synthesis and lipoteichoic acid anchoring in Staphylococcus aureus. J. Bacteriol. 189, 2521-2530.

Gründling, A., and Schneewind, O. (2007b) Synthesis of glycerol phosphate lipoteichoic acid in Staphylococcus aureus. Proc. Natl. Acad. Sci. USA 104, 8478-8483.

Guariglia-Oropeza, V., and Helmann, J. D. (2011) Bacillus subtilis $\sigma^{\mathrm{V}}$ confers lysozyme resistance by activation of two cell wall modification pathways, peptidoglycan O-acetylation and D-alanylation of teichoic acids. J. Bacteriol. 193, 62236232.

Hachmann, A. B., Sevim, E., Gaballa, A., Popham, D. L., Antelmann, H., and Helmann, J. D. (2011) Reduction in membrane phosphatidylglycerol content leads to daptomycin resistance in Bacillus subtilis. Antimicrob. Agents Chemother. 55, 4326-4337.

Hashimoto, M., Seki, T., Matsuoka, S., Hara, H., Asai, K., Sadaie, Y., and Matsumoto, K. (2013) Induction of extracytoplasmic function sigma factors in Bacillus subtilis cells with defects in lipoteichoic acid synthesis. Microbiology 159, 23-35.

Hashimoto, M., Takahashi, H., Hara, Y., Hara, H., Asai, K., Sadaie, Y., and Matsumoto, K. (2009) Induction of extracytoplasmic function sigma factors in Bacillus subtilis cells with membranes of reduced phosphatidylglycerol content. Genes Genet. Syst. 84, 191-198.
Hastie, J. L., Williams, K. B., Bohr, L. L., Houtman, J. C., Gakhar, L., and Ellermeier, C. D. (2016) The anti-sigma factor RsiV is a bacterial receptor for lysozyme: co-crystal structure determination and demonstration that binding of lysozyme to $\mathrm{RsiV}$ is required for $\sigma^{\mathrm{V}}$ activation. PLoS Genet. 12, e1006287.

Hastie, J. L., Williams, K. B., and Ellermeier, C. D. (2013) The activity of $\sigma^{\mathrm{V}}$, an extracytoplasmic function $\sigma$ factor of Bacillus subtilis, is controlled by regulated proteolysis of the anti- $\sigma$ factor RsiV. J. Bacteriol. 195, 3135-3144.

Hastie, J. L., Williams, K. B., Sepulveda, C., Houtman, J. C., Forest, K. T., and Ellermeier, C. D. (2014) Evidence of a bacterial receptor for lysozyme: binding of lysozyme to the anti- $\sigma$ factor RsiV controls activation of the ecf $\sigma$ factor $\sigma^{\mathrm{V}}$. PLoS Genet. 10, e1004643.

Heinrich, J., Hein, K., and Wiegert, T. (2009) Two proteolytic modules are involved in regulated intramembrane proteolysis of Bacillus subtilis RsiW. Mol. Microbiol. 74, 14121426.

Helmann, J. D. (2002) The extracytoplasmic function (ECF) sigma factors. Adv. Microb. Physiol. 46, 47-110.

Ho, T. D., Hastie, J. L., Intile, P. J., and Ellermeier, C. D. (2011) The Bacillus subtilis extracytoplasmic function $\sigma$ factor $\sigma^{\mathrm{V}}$ is induced by lysozyme and provides resistance to lysozyme. J. Bacteriol. 193, 6215-6222.

Jorasch, P., Wolter, F. P., Zahringer, U., and Heinz, E. (1998) A UDP glucosyltransferase from Bacillus subtilis successively transfers up to four glucose residues to 1,2-diacylglycerol: expression of ypfP in Escherichia coli and structural analysis of its reaction products. Mol. Microbiol. 29, 419-430.

Kawai, F., Shoda, M., Harashima, R., Sadaie, Y., Hara, H., and Matsumoto, K. (2004) Cardiolipin domains in Bacillus subtilis marburg membranes. J. Bacteriol. 186, 1475-1483.

Kikuchi, S., Shibuya, I., and Matsumoto, K. (2000) Viability of an Escherichia coli pgsA null mutant lacking detectable phosphatidylglycerol and cardiolipin. J. Bacteriol. 182, 371-376.

Kiriukhin, M. Y., Debabov, D. V., Shinabarger, D. L., and Neuhaus, F. C. (2001) Biosynthesis of the glycolipid anchor in lipoteichoic acid of Staphylococcus aureus RN4220: role of YpfP, the diglucosyldiacylglycerol synthase. J. Bacteriol. 183, 3506-3514.

Luo, Y., and Helmann, J. D. (2012) Analysis of the role of Bacillus subtilis $\sigma^{\mathrm{M}}$ in $\beta$-lactam resistance reveals an essential role for c-di-AMP in peptidoglycan homeostasis. Mol. Microbiol. 83, 623-639.

Matsumoto, K., Hara, H., Fishov, I., Mileykovskaya, E., and Norris, V. (2015) The membrane: transertion as an organizing principle in membrane heterogeneity. Front. Microbiol. fmicb.2015.00572.

Matsuoka, S., Chiba, M., Tanimura, Y., Hashimoto, M., Hara, H., and Matsumoto, K. (2011) Abnormal morphology of Bacillus subtilis ugtP mutant cells lacking glucolipids. Genes Genet. Syst. 86, 295-304.

Matsuoka, S., Seki, T., Matsumoto, K., and Hara, H. (2016) Suppression of abnormal morphology and extracytoplasmic function sigma activity in Bacillus subtilis ugtP mutant cells by expression of heterologous glucolipid synthases from Acholeplasma laidlawii. Biosci. Biotechnol. Biochem. 80, 2325-33.

Miller, J. H. (1992) A Short Course in Bacterial Genetics: A Laboratory Manual and Handbook for Escherichia coli and Related Bacteria. Cold Spring Harbor Laboratory Press, New York, USA.

Murray, E. J., and Stanley-Wall, N. R. (2010) The sensitivity 
of Bacillus subtilis to diverse antimicrobial compounds is influenced by Abh. Arch. Microbiol. 192, 1059-1067.

Neuhaus, F. C., and Baddiley, J. (2003) A continuum of anionic charge: structures and functions of D-alanyl-teichoic acids in gram-positive bacteria. Microbiol. Mol. Biol. Rev. 67, 686-723.

Petersohn, A., Brigulla, M., Haas, S., Hoheisel, J. D., Völker, U., and Hecker, M. (2001) Global analysis of the general stress response of Bacillus subtilis. J. Bacteriol. 183, 5617-5631.

Reichmann, N. T., and Grundling, A. (2011) Location, synthesis and function of glycolipids and polyglycerolphosphate lipoteichoic acid in Gram-positive bacteria of the phylum Firmicutes. FEMS Microbiol. Lett. 319, 97-105.

Salzberg, L. I., and Helmann, J. D. (2008) Phenotypic and transcriptomic characterization of Bacillus subtilis mutants with grossly altered membrane composition. J. Bacteriol. 190, 7797-7807.

Sambrook, J., and Russell, D. W. (2001) Molecular Cloning: A Laboratory Manual, 3rd edition, Cold Spring Horbor laboratory Press, New York, USA.

Schirner, K., Marles-Wright, J., Lewis, R. J., and Errington, J. (2009) Distinct and essential morphogenic functions for wall- and lipo-teichoic acids in Bacillus subtilis. EMBO J. 28, 830-842.

Schobel, S., Zellmeier, S., Schumann, W., and Wiegert, T. (2004) The Bacillus subtilis $\sigma^{\mathrm{W}}$ anti-sigma factor RsiW is degraded by intramembrane proteolysis through YluC. Mol. Microbiol. 52, 1091-1105.
Seki, T., Matsumoto, K., Matsuoka, S., and Hara, H. (2017) Activation without proteolysis of anti- $\sigma$ factor $\mathrm{RsiV}$ of the extracytoplasmic function $\sigma$ factor $\sigma^{\mathrm{V}}$ in a glucolipid-deficient mutant of Bacillus subtilis. Adv. Microbiol. 7, 315-327.

Seki, T., Mineshima, R., Hashimoto, M., Matsumoto, K., Hara, H., and Matsuoka, S. (2015) Repression of the activities of two extracytoplasmic function $\sigma$ factors, $\sigma^{\mathrm{M}}$ and $\sigma^{\mathrm{V}}$, of Bacillus subtilis by glucolipids in Escherichia coli cells. Genes Genet. Syst. 90, 109-114.

Suzuki, M., Hara, H., and Matsumoto, K. (2002) Envelope disorder of Escherichia coli cells lacking phosphatidylglycerol. J. Bacteriol. 184, 5418-5425.

Thackray, P. D., and Moir, A. (2003) SigM, an extracytoplasmic function sigma factor of Bacillus subtilis, is activated in response to cell wall antibiotics, ethanol, heat, acid, and superoxide stress. J. Bacteriol. 185, 3491-3498.

Wang, P. Z., and Doi, R. H. (1984) Overlapping promoters transcribed by Bacillus subtilis $\sigma^{55}$ and $\sigma^{37}$ RNA polymerase holoenzymes during growth and stationary phases. J. Biol. Chem. 259, 8619-8625.

Wörmann, M. E., Corrigan, R. M., Simpson, P. J., Matthews, S. J., and Gründling, A. (2011) Enzymatic activities and functional interdependencies of Bacillus subtilis lipoteichoic acid synthesis enzymes. Mol. Microbiol. 79, 566-583.

Zellmeier, S., Schumann, W., and Wiegert, T. (2006) Involvement of Clp protease activity in modulating the Bacillus subtilis $\sigma^{\mathrm{W}}$ stress response. Mol. Microbiol. 61, 1569-1582. 